\title{
Resistência de cultivares de alfafa à antracnose e à mancha de leptosphaerulina em Uberlândia-MG
}

\author{
Fernando César Juliatti ${ }^{1,2}$, Fernando Alves Pimenta ${ }^{1}$, Juliana Araújo Santos Martins ${ }^{1}$, Edson Ampélio Pozza ${ }^{3}$, \\ Sandomar de Angelo da Silva ${ }^{1}$, Maristela dos Santos Rey ${ }^{1}$, Roberto Resende dos Santos ${ }^{1}$
}

\begin{abstract}
${ }^{1}$ Universidade Federal de Uberlândia, LAMIP (Laboratório de Micologia e Proteção de Plantas), Instituto de Ciências Agrárias, Núcleo de Fitopatologia, Av. Amazonas s/no, Campus Umuarama, Bloco 2E, CEP 38400-920 Uberlândia, MG. ${ }^{2}$ Professor Titular do Departamento de Fitopatologia da UFU. ${ }^{3}$ Departamento de Fitopatologia, Universidade Federal de Lavras, CP 37, CEP 37200-000, Lavras, MG.

Autor para correspondência: Fernando César Juliatti (juliatti@ufu.br)
\end{abstract}

Data de chegada: 21/10/2009. Aceito para publicação em: 10/10/2011.

\section{RESUMO}

Juliatti, F.C.; Pimenta, F. A.; Martins, J. A. S.; Pozza, E. A.; Silva, S. A.; Rey, M.S.; Santos, R.R. Resistência de cultivares de alfafa à Antracnose e à Mancha de Leptosphaerulina em Uberlândia-MG. Summa Phytopathologica, v.37, n.4, p.169-173, 2011.

Tendo em vista a importância da alfafa como forrageira para a produção de feno de alta qualidade, avaliou-se a susceptibilidade de 27 cultivares às doenças de parte aérea bem como progresso da doença nas condições climáticas da região de Uberlândia-MG. As doenças diagnosticadas foram: antracnose (Colletotrichum trifolii Bain \& Essary) e mancha de leptosferulina (Leptosphaerulina briosiana (Pollaci) J. H. Graham \& Luttrel). Os resultados indicaram as cultivares BR1, MONARCA, SUTTER, MARICOPA,
BR4，SW8210，5929 e MH15 mais resistentes às lesões de leptosferulina. As cultivares ARAUCANA, IC1990, MH15 E SW8112A mostraram-se resistentes à antracnose. As cultivares Maricopa e ICI990 não apresentaram desfolha. O trabalho apresentou uma correlação simples significativa entre severidade da antracnose e porcentagem de desfolha. O modelo quadrático adequou-se para a curva de progresso da doença da mancha de leptosferulina.

Palavras-chave adicionais: Medicago sativa L., Leptosphaerulina briosiana, Colletotrichum trifolii, AACPD, desfolha.

\section{ABSTRACT}

Juliatti, F. C.; Pimenta, F. A.; Martins, J. A. S.; Pozza, E. A.; Silva, S. A. ; Rey, M.S.; Santos, R.R. Resistance alfalfa cultivars to Antracnosis and Leptosphaerulina leaf spot in Uberlândia-MG. Summa Phytopathologica, v.37, n.4, p.169-173, 2011.

In view of the importance of alfalfa as fodder for the production of high quality hay, it was evaluated the susceptibility of 27 cultivars to diseases of the shoot as well as progress of the disease in the region of Uberlândia-MG. The diseases were diagnosed: anthracnose (Colletotrichum trifolii Bain \& Essary) and Leptosphaerulina leaf spot (Leptosphaerulina briosiana (Pollaci) JH Graham \& Luttrel). The results showed that cultivars BR1, MONARCA, SUTTER,
MARICOPA, BR4, SW821O, 5929 and MH15 more resistant to damage from leptosferulina. Cultivars ARAUCANA, IC1990, MH15 and SW8112A were resistant to anthracnose. Cultivars Maricopa is not presented ICI990 defoliation. The study showed a significant simple correlation between severity of anthracnose and percentage of defoliation. During the adjustment of the disease progress curves for the Leptosphaerulina leaf spot, the model adjusted was the square.

Keywords: Medicago sativa L., Leptosphaerulina briosiana, Colletotrichum trifolii, AACPD, defoliation.

A alfafa (Medicago sativa L.) é uma leguminosa forrageira perene, rica em proteínas, cálcio, fósforo e vitaminas Ae C (12), tenra, suculenta e muito palatável. A alfafa reúne características desejáveis para plantas forrageiras, entre as quais: (a) alto valor nutritivo - contém aproximadamente duas vezes mais proteína digestível do que o trevo (Trifolium repens) e quatro vezes mais do que a silagem de milho (12); (b) produz grandes quantidades de matéria verde, com excelente palatabilidade e digestibilidade. Por essas características, entre outras, é considerada por muitos, como a mais importante das forrageiras cultivadas (3). Com a implantação do sistema intensivo de criação de gado para produção de leite na região sudeste do Brasil, aumentou a demanda de alimentos volumosos de alto valor nutritivo para melhor aproveitamento do padrão genético dos animais (11).
No Brasil, as pragas e doenças que ocorrem na alfafa são ainda praticamente desconhecidas, bem como os danos causados. Os países que se dedicam intensivamente ao cultivo desta forrageira estudam exaustivamente estes organismos, sendo inclusive, um dos aspectos mais enfatizados na seleção e no desenvolvimento de cultivares (14).

O próprio manejo da cultura da alfafa proporciona o desenvolvimento de doenças, principalmente as operações de cortes, enleiramentos, recolhimento e controle de plantas daninhas. Todas essas práticas culturais podem causar injúrias às raízes e à coroa, o que facilita a penetração dos patógenos, além da sua disseminação. Assim, em alfafais mecanizados, há uma tendência do aparecimento de mais doenças (4).

No sul de Minas Gerais, onde o cultivo de alfafa aumentou 
significativamente em decorrência da grande demanda por feno, para criação de cavalos e bezerros, diversas doenças foram constatadas, com destaque para a antracnose (Colletotrichum frifolli Bain \& Essary), mancha foliar e talo negro de verão (Cercospora medicaginis Ellis \& Everch), ferrugem (Uromyces striatus J. Schrot) e mancha de leptosferulina (Leptosphaerulina briosiana (Pollacci) J. H. Graham \& Lutrell) (15).

Em períodos chuvosos e quentes, de uma maneira geral, as doenças sempre são mais abundantes e as lesões se desenvolvem mais rapidamente em número e tamanho, levando à desfolha da planta (17). Os patógenos podem sobreviver em folhas de plantas hospedeiras e se multiplicarem sob condições de baixa umidade ou ambiente desfavorável, sem causar sintomas aparentes da doença e as epidemias ocorrem quando as condições do ambiente tornam-se favoráveis (19).

A antracnose (Colletotrichum trifolii Bain \& Essay) é considerada uma das mais importantes doenças da alfafa, causando em infecções severas, perdas de 25 a $30 \%$ da produção e do "stand" (19), podendo formar com outras doenças o complexo chamado de "haste negra". Os sintomas da antracnose se caracterizam pela ocorrência de lesões necróticas no caule, alongadas, com centro escuro correspondente às frutificações do fungo. Os sintomas que correspondem aos problemas maiores da antracnose são as podridões do colo, que ao envolver a circunferência completa do mesmo, mata a planta (17).

Segundo LEATH (7), o agente causador da mancha de leptosferulina é o fungo Leptosphaerulina briosiana (Pollacci) J. G. Graham \& Luttrell. A mancha de leptosferulina inicialmente ataca as folhas novas, podendo infectar também folhas mais velhas e pecíolos. As lesões possuem os bordos marrom-escuros com centro claro e halos c1oróticos. Quando as condições ambientais são favoráveis, as lesões são maiores e se unem formando o que se conhece como queima das folhas. Como conseqüência do da alta severidade ocorre a queda das folhas (18). O controle da mancha de leptosferulina é alcançado pelo uso de cultivares resistentes (10).

Assim, este trabalho objetivou avaliar a resistência de 27 cultivares de alfafa à manchas foliares de leptosferulina e a antracnose, descrever as epidemias e correlacionar as doenças com a produção de matéria seca no período de janeiro a junho nas condições de Uberlândia-MG.

\section{MATERIAL E MÉTODOS}

O experimento foi realizado na fazenda do Glória, da Universidade Federal de Uberlândia (UFU). O clima da região segundo a classificação de Koppen, adotada universalmente, é do tipo "Cwa", sendo didaticamente classificado como Tropical de Altitude.

Avaliaram-se 27 cultivares de alfafa (Medicago sativa L.) utilizando-se o delineamento experimental de blocos casualizados com três repetições. As parcelas, não foram separadas entre si, eram constituídas de 5 fileiras de $5 \mathrm{~m}$ de comprimento espaçadas de $30 \mathrm{~cm}$, ou seja, $5 \mathrm{~m}$ de comprimento por $1,5 \mathrm{~m}$ de largura. Os blocos eram separados por corredores de 1,50 m. Quando foi efetuado o corte descartaram-se as duas linhas laterais, $1 \mathrm{~m}$ na cabeceira e $1 \mathrm{~m}$ no fundo, totalizando uma área útil de $2,70 \mathrm{~m}^{2}$.

Foi utilizado sistema de irrigação por aspersão quando necessário. Como adubação de reposição aplicou-se $15 \mathrm{~kg}$ de super simples, 3,5 $\mathrm{kg}$ de cloreto de potássio e $210 \mathrm{~kg}$ de esterco de galinha por bloco.

Foram realizadas avaliações mensais, antes dos cortes, para determinação da porcentagem de desfolha e curva de progresso das doenças, no período de janeiro a junho de 1996, das seguintes cultivares: BR2, 5888, SEMIT 921, ALFA 200, ELGRANDE, 5929, MARICOPA, MONARCA, BR4, P 30, ALTO, 22 SUTTER, SW 9210A, WL 516, FALCOM, BR3, FLORIDA, MH4, MH15, VALPLUS, ARAUCANA, SW 8112 A, SW 8210, BR 1, RIO, ICI 990, 5715, as quais fazem parte do projeto CIAT-EMBRAPA-UFU, visando-se observar cultivares mais adaptadas às condições edafoclimáticas, da região de Uberlândia, MG.

O reconhecimento e identificação das doenças foram realizados no laboratório de Micologia e Proteção de Plantas da UFU (LAMIP), preliminarmente pelo quadro sintomatológico e confirmado segundo a morfologia do patógeno em microscópio óptico com aumento de 400 vezes, após incubação a 25 graus Celsius, por 7 dias, sob luz do dia alternada com o escuro em $12 \mathrm{~h}$.

Avaliou-se a severidade da mancha de leptosferulina (Lepfosphaelina briosiana (Poliacci) J. H. Grahan \& Luttrel) observando-se em cada uma das dez hastes coletadas por parcela, a quinta folha a partir do ápice, contando-se o número de lesões nessa folha. Adotou-se a porcentagem de área foliar doente de 0 a $100 \%$, conforme discriminado na Figura 1. Para determinação da área lesionada da haste com antracnose (Colletotrichum trifolii Bain \& Essary) quantificou-se a porcentagem da área de 10 caules doentes com haste negras em relação ao total de hastes padronizadas para $10 \mathrm{~cm}$. Esta avaliação considerou-se a porcentagem de tecido doente em relação ao comprimento total do caule.

As folhas e talos de toda parte aérea com mais de $30 \mathrm{~cm}$ foram cortados com estilete, colhidas em cada parcela, nos três blocos, foram pesados imediatamente em uma balança, para estimativa da produção de matéria verde. Depois de pesados, o material de cada cultivar era misturado e então retirado uma amostra composta, de $500 \mathrm{~g}$ em saco de papel. As amostras foram colocadas em estufa a $65^{\circ} \mathrm{C}$ por 72 horas, para determinação de matéria seca.

Para avaliação de desfolha, seguiu-se a metodologia descrita por THAL \& CAMPBELL (19), que consiste em coletar, de modo aleatório,
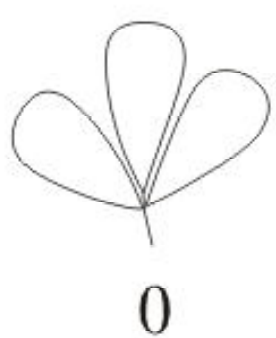

$0 \%$

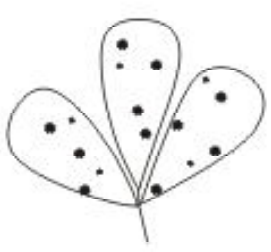

1

$10 \%$

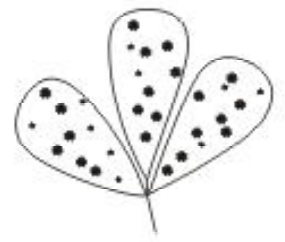

2

$30 \%$

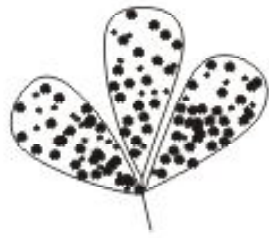

3

$60 \%$

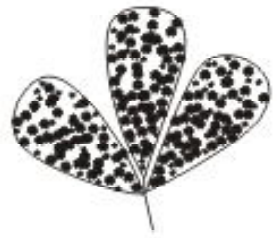

4

$80 \%$

Figura 1. Escala diagramática usada para estimativa da porcentagem de área foliar lesionada em alfafa. 
dez hastes por parcela e determinar a porcentagem de nós desfolhados segundo a fórmula: [(número de nós desfolhados / número total de nós da planta) x 100].

Foram plotadas as curvas de progresso da doença para todas as cultivares considerando-se os dados médios das três repetições. Com esses dados, estimaram-se, ainda, a área abaixo da curva de progresso da doença (AACPD), que é usada para observar uma epidemia. No cálculo da área abaixo da curva de progresso da doença, utilizou-se a equação proposta por SHANNER \& FINLEY (15). A AACPD foi padronizada, ao dividir o valor da área abaixo da curva de progresso pela duração do tempo total (tn- $t_{1}$ ) da epidemia (2), para comparar epidemias de diferentes durações. Os dados de AACPD foram submetidos à analise estatística, sendo as médias comparadas pelo teste Scott Knott, ao nível de 5\% de probabilidade.Onde:

$$
\begin{aligned}
& \text { n-1 } \\
& \mathrm{AACPD}=\Sigma(\mathrm{Yi}+1) / 2 \mathrm{x}(\mathrm{Ti}+1-\mathrm{T} 1) \\
& \text { i-1 }
\end{aligned}
$$

Em que:

$Y i=$ proporção da doença na $\mathrm{i}$ - ésima observação
Ti $=$ Tempo (dias) na i-ésima observação

$\mathrm{n}=$ número total de observações

Foram realizadas correlações entre produção de matéria seca, índice de desfolha e severidade das doenças (antracnose e mancha de leptosferulina).

Os ajustes das curvas de progresso da doença foram realizados para leptosferulina, segundo os modelos gompertz, logarimithmic, logistic, monomolecular, linear e quadrático. Para escolha do melhor modelo empregou-se como critério de avaliação, o coeficiente de determinação $\left(\mathrm{R}^{2}\right)$ e o quadrado médio do desvio (QMD) (6).

\section{RESULTADOS E DISCUSSÃO}

De acordo com a análise estatística para a ACPD da mancha de leptosferulina pelo teste de Scott-Knott (Tabela 01), constatou-se que a cultivar ELGRANDE teve maior AACPD, sendo então, a que apresentou maior suscetibilidade às lesões foliares causadas pela referida doença. Em contraposto, as cultivares BR1, MONARCA, SUTTER, MARICOPA, BR4, SW8210, 5929 e MH15 apresentaram menores AACPD, mostrando-se mais resistentes a essa doença. A

Tabela 1. Área abaixo da curva de progresso de doença (A.A.C.P.D.) para a severidade da mancha de Leptosphaerulina (S.M.L.), e da Antracnose nos ramos (S.L.A.R.), porcentagem de nós desfolhados (P.N.D.), Matéria Verde (M.V) e Matéria Seca (M.S.) em 27 cultivares de alfafa, Uberlândia - MG.

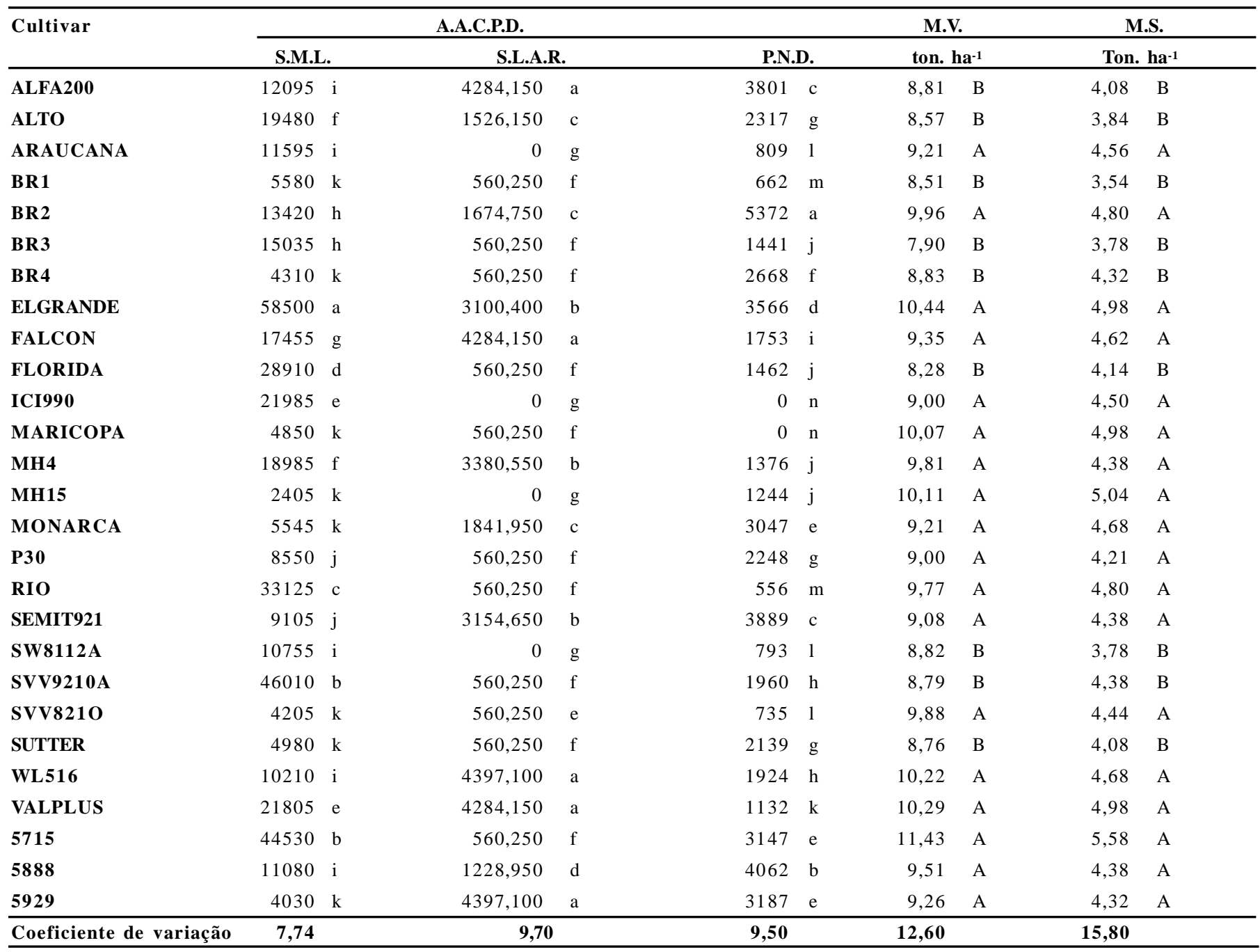

*Médias seguidas por mesmas letras não diferem entre si pelo teste de Scott Knott ao nível de 5\% de probabilidade. 
cultivar MH15 foi a que apresentou menor AACPD dentre todas, apesar de não diferir estatisticamente das cultivares supracitadas.

Analisando-se a AACPD da área lesionada da haste com antracnose (Tabela 01), observou-se que as cultivares 5929, WL516, VALPLUS, FALCON e ALFA200 obtiveram altos níveis de severidade de antracnose. Entretanto, as cultivares VALPUS, FALCON, e WL516 podem ser consideradas tolerantes, já que produziram altas taxas de matéria seca, mesmo sob o ataque da doença. As cultivares SW8112, MH15, ICI990 e ARAUCANA apresentaram resistência, ou seja, à AACPD foi nula (zero de doença). Já BOTREL et al. (1) em experimento realizado, mostraram que as cultivares ICI 990 e ARAUCANA, mostraram-se com reações de suscetibilidade e baixa resistência à doenças, respectivamente, em época de alta precipitação. O mesmo autor ainda sugere que as cultivares FALCON e WL516 mostram-se com reação de baixa resistência e resistente, respectivamente.

Pelo comportamento da cultivar MH15 em relação à Mancha de leptosferulina e antracnose seria viável a utilização deste material como fonte de resistência em programas de melhoramento, visto que em trabalho realizado por PEREIRA et al. (13), esta cultivar também destacou-se também por obter uma reação de resistência a ferrugem.

Em estudo realizado por THAL \& CAMPBELL (19), observaram que a desfolha não é decorrente somente do alto índice de doenças foliares; outros fatores também são sugeridos como, estádio de desenvolvimento fisiológico das plantas, variabilidade genética e variáveis climáticas e período de seca. Neste estudo os maiores valores de precipitação, umidade relativa e temperatura foram observados nos meses de janeiro, fevereiro e março (Tabela 2).

O estádio de crescimento da alfafa tem que ser levado em consideração na avaliação das doenças de parte aérea. A queda das folhas no momento que antecede a colheita é um fenômeno comum e variável entre cultivares diferentes (19).

O teste para variável porcentagem de desfolha (Tabela 01) mostra a maior susceptibilidade da cultivar BR2 a desfolha, e a maior capacidade de persistência das folhas nas cultivares MARICOPA e ICI990, porém estas mostraram-se indiferentes estatisticamente de

Tabela 2. Dados climáticos de temperatura, precipitação total e umidade relativa na cidade de Uberlândia - MG, no período de janeiro a junho (Médias mensais).

\begin{tabular}{lccc}
\hline & $\begin{array}{c}\text { Precipitação } \\
(\mathbf{m m})\end{array}$ & $\begin{array}{c}\text { Umidade relativa } \\
(\boldsymbol{\%})\end{array}$ & $\begin{array}{c}\text { Temperatura } \\
{ }^{\mathbf{0}} \mathbf{C}\end{array}$ \\
\hline JANEIRO & 208,3 & 68 & 24,5 \\
FEVEREIRO & 189,5 & 75 & 24,2 \\
MARÇO & 258,1 & 81 & 23,6 \\
ABRIL & 96,5 & 75 & 23,1 \\
MAIO & 6,4 & 64 & 20 \\
JUNHO & 1,5 & 62 & 19,8 \\
\hline
\end{tabular}

BR1 e RIO. Estes dados discordam de PERREIRA et al. (13), na qual os autores em um estudo de resistência de cultivares, na mesma região evidenciaram uma alta desfolha da cultivar MARICOPA, sob o ataque de doenças foliares.

Analisando-se os coeficientes de correlação simples (Tabela 03) entre as variáveis antracnose, desfolha, matéria seca e mancha de leptosferulina observou-se que houve correlação significativa apenas entre antracnose e desfolha, mostrando que esta doença influenciou decisivamente nos meses de janeiro a junho onde ocorreu a maior porcentagem de desfolha. Resultados semelhantes foram obtidos por BOTREL et al. (1), em experimento conduzido em área de Mata Atlântica, também no estado de Minas Gerais, onde a incidência de doenças na alfafa foi mais freqüente durante o período das chuvas,

Tabela 4. Modelos de progresso da doença (M.P.D) e coeficientes de determinação $\left(R^{2}\right)$ para as cultivares de alfafa estudadas.

\begin{tabular}{lcc}
\hline Cultivares & M. P.D. & $\mathbf{R}^{2}$ \\
\hline ALFA200 & $-3,0954 \mathrm{x}^{2}+21,514 \mathrm{x}+44,202$ & 0,86 \\
ALTO & $-3,1843 \mathrm{x}^{2}+19,537 \mathrm{x}+95,97$ & 0,82 \\
ARAUCANA. & $-3,7616 \mathrm{x}^{2}+22,93 \mathrm{x}+45,073$ & 0,87 \\
BR1 & $-2,0837 \mathrm{x}^{2}+12,653 \mathrm{x}+20,263$ & 0,90 \\
BR2 & $-1,6543 \mathrm{x}^{2}+9,552 \mathrm{x}+72,138$ & 0,92 \\
BR3 & $-2,4766 \mathrm{x}+98,89$ & 0,87 \\
BR4 & $-2,5239 \mathrm{x}^{2}+16,324 \mathrm{x}+6,532$ & 0,89 \\
ELGRANDE & $-7,4164 \mathrm{x}^{2}+41,554 \mathrm{x}+317,6$ & 0,90 \\
FALCON & $-3,0355 \mathrm{x}^{2}+16,305 \mathrm{x}+105,14$ & 0,89 \\
FLÓRIDA & $-4,8741 \mathrm{x}^{2}+27,729 \mathrm{x}+149,71$ & 0,90 \\
ICI990 & $-0,3454 \mathrm{x}^{2}-1,2585 \mathrm{x}+141,87$ & 0,93 \\
MARICOPA & $-1,6957 \mathrm{x}^{2}+9,8138 \mathrm{x}+20,041$ & 0,89 \\
MH4 & $-2,5657 \mathrm{x}^{2}+15,17 \mathrm{x}+99,7666$ & 0,87 \\
MH15 & $-1,8284 \mathrm{x}^{2}+11,741 \mathrm{x}+0,691$ & 0,91 \\
MONARCA & $-2,0357 \mathrm{x}^{2}+12,745 \mathrm{x}+17,268$ & 0,91 \\
P30 & $-2,72 \mathrm{x}^{2}+15,897 \mathrm{x}+36,17$ & 0,86 \\
RIO & $-3,1725 \mathrm{x}^{2}+16,217 \mathrm{x}+189,97$ & 0,90 \\
SEMIT921 & $-3,8693 \mathrm{x}^{2}+24,428 \mathrm{x}+27,13$ & 0,88 \\
SW9210A & $-3,0243 \mathrm{x}^{2}+16,151 \mathrm{x}+266,06$ & 0,85 \\
SW8210 & $-2,7254 \mathrm{x}^{2}+17,725 \mathrm{x}+4,074$ & 0,80 \\
SUTTER & $-2,0539 \mathrm{x}^{2}+12,27 \mathrm{x}+17,028$ & 0,89 \\
SW8112A & $-2,1129 \mathrm{x}^{2}+13,219 \mathrm{x}+17,028$ & 0,89 \\
WL516 & $-4,6071 \mathrm{x}^{2}+28,488 \mathrm{x}+30,332$ & 0,93 \\
VALPLUS & $-4,2028 \mathrm{x}^{2}+25,26 \mathrm{x}+105,70$ & 0,98 \\
5715 & $-1,697 \mathrm{x}^{2}+7,7371 \mathrm{x}+267,16$ & \\
5888 & $-3,9046 \mathrm{x}^{2}+24,589 \mathrm{x}+39,268$ & \\
5929 & $-1,4752 \mathrm{x}^{2}+9,212 \mathrm{x}+14,075$ & \\
\hline & & \\
& &
\end{tabular}

Tabela 3. Coeficiente de correlação simples entre mancha de leptosferulina, antracnose, porcentagem do caule desfolhado e matéria seca.

\begin{tabular}{lllll}
\hline Nome & Nome & Valor t & & Prob. $>$ t \\
\hline Leptosferulina & Matéria seca & 1,4349 & 0,160544 & $\mathrm{Ns}$ \\
Leptosferulina & Desfolha & 0,4575 & 0,655455 & $\mathrm{Ns}$ \\
Leptosferulina & Antracnose & 0,3038 & 0,761247 & $\mathrm{Ns}$ \\
Desfolha & Matéria seca & 0,4405 & 0,666981 & $\mathrm{Ns}$ \\
Desfolha & Antracnose & 2,3819 & 0,023775 & $*$ \\
Antracnose & Matéria seca & 0,6 & 0,560505 & $\mathrm{Ns}$ \\
\hline
\end{tabular}


provavelmente devido às condições climáticas predominantes nessa época do ano (temperatura e umidade elevadas), que são favoráveis ao desenvolvimento das principais doenças da alfafa (9).

Os respectivos modelos de progresso da doença para as 27 cultivares encontram-se na Tabela 04.O modelo quadrático foi o que melhor ajustou às curvas de progresso da doença para mancha de leptosferulina, para as 27 cultivares.

Dentre os principais resultados, do presente trabalho, pode-se listar:

1. Houve correlação significativa entre a severidade da antracnose, avaliado pela desfolha;

2. Para avaliação de desfolha, devem-se levar em consideração outros fatores como idade fisiológica das plantas, microclima e variabilidade genética;

3. As cultivares MARICOPA, ICI990, RIO e BR1 mostraram-se resistentes à desfolha;

4. As cultivares BR1, MONARCA, SUTTER, MARICOPA, BR4, SW8210 e MH15, foram as que apresentaram menor AACPD em relação às lesões de leptosferulina;

5. As cultivares SW8112A, MH15, ICI990 e ARAUCANA foram resistentes à antracnose.

6. O modelo quadrático é o que melhor se ajusta às curvas de progresso da doença para a mancha de leptosferulina.

\section{REFERÊNCIAS BIBLIOGRÁFICAS}

1. Botrel, M. de A.; Ferreira, R. de P.; Alvim, M. J.; Xavier, D. F. Cultivares de alfafa em área de influência da Mata Atlântica no Estado de Minas Gerais. Pesquisa Agropec. Brasileira, Brasília vol.36 no.11, nov. 2001.

2. Fry, W.E. Integrated control of potatoes late blight: effects of polygenic resistance and techniques of timing fungicide application. Phytopathology, St Paul, v. 67, p.415-420, 1977.

3. Gomes, F. T. Comportamento da Alfafa Inoculada com Rizóbio, em Resposta a Níveis de Corretivo, com Diferentes Relações Cálcio:Magnésio. Viçosa: UFV. 1995. 87p. (Dissertação, Mestrado em Microbiologia Agrícola).4. Honda, C.S., Honda, A.M. Cultura da alfafa. Cambará : IARA, 1990. 245p.

5. Iamauti, M.T.; Salgado, C.L. Doenças da alfafa. In: Kimati, H.; Amorin, L.; Bergamim Filho, A. et al. (Eds.). Manual de fitopatologia: doenças das plantas cultivadas. 2 ed. São Paulo:
Agronômica Ceres, 1997. v.2, p.26-32.

6. Irwin, J.A.G. Factors contributing to poor lucerne persistence in southern Queensland. Aust. J. Exp. Agric. Anim. Husb. 17, 998-1003. 1977.

7. Jesus Junior, W. C.; Pozza, E.A.; Valle, F.X.R.; Aguilero, G.M. Análise Temporal de Epidemias. In: Valle, F.X.R.; Jesus Júnior, W.C.; Zambolim, L. Epidemiologia aplicada ao manejo de doenças de plantas. Belo Horizonte: Perffil, 2004, cap. 4, p.127.

8. Leath, K. T. Leptosphaerulina leaf spot. In: Stuteville, D.L.; Erwin, D.C. Compendium of Alfalfa Diseases. 2.ed. St. Paul: APS Press, 1990. p. 15.

9. Melton, B.; Bountray, J. B.; Bouton, J. H. Geographic adaptation and cultivar selection. In: Hanson, H. A.; Harnes, H. K.; Hill, R. R. Alfalfa and alfalfa improvement. Madison: American Society of Agronomy, 1988. p. 596-618

10. Monteiro, A. L. G. ; Valério, M. A. ; Whitaker, H. M. A. . Avaliação de dezesseis cultivares de alfafa (Medicago sativa L.) em Bandeirantes-PR. Anais da Sociedade Brasileira de Zootecnia, Piracicaba, v.30, p. 3, 1993.

11. Nuernberg, N.J., Milan, P.A., Silveira, C.A.M. Cultivo, manejo e utilização da alfafa. In: Nuernberg, N.J., Milan, P.A., Silveira, C.A.M. Manual de produção de alfafa. Florianópolis : EMPASC, 1990. p.15-56.

12. Oliveira, P. R. D. Avaliação da produção e da qualidade de cultivares de alfafa (Medicago sativa $\mathrm{L}$.). Piracicaba: ESALGUSP, 1986. 67 p. (Tese - Mestrado em Zootecnia).

13. Pereira, R. C.; Evangelista, A. R.; Souza, P. E.; Silva, A. C.; Muniz, J. A. Doenças Foliares da Alfafa (Medicago sativa L.), em Lavras, Minas Gerais. Revista Bras. Zootecnia, v.32, n.6, p.1287-1296, 2003.

14. Pozza, E. A.; Souza, P. E. Ocorrência de Doenças em Alfafa (Medicago sativa L.) na região de Lavras, MG. Ciência e Prática, Lavras, v. 18, n. 2, p. 186-188, 1994.

15. Shaner, G.; Finley, R..F. The effects of nitrogen fertilization on the expression of slow-mildewing in know wheat. Phytopathology, St. Paul, v.70, p. 1183-86, 1977.

16. Souza, P. E. de. Epidemiologia e controle das manchas foliares da alfafa (Medicago sativa L.). Lavras: Universidade Federal de Lavras, 1996. 56 p. (Tese) Doutorado.

17. Stuteville, D.L Rust. In: Stuteville, D.L.; Erwin, D.C. Compendium of Alfalfa Diseases. 2.ed. St. Paul: APS Press, 1990. p. 15 .

18. Stuteville, D.L.; Erwin, D.C. Compendium of Alfalfa Diseases. 2.ed. St. Paul: APS Press, 1990.84p.

19. Thal, W. M., Campbell, C. L. Assessment of resistance to leaf spot diseases among alfafa cultivas in North Carolina fields. Phytopathology, St. Paul, v. 77, n. 6, p. 964-966, June. 1987. 